\title{
CARACTERIZACIÓN GEOLÓGICO-ESTRUCTURAL DE ALGUNAS ZONAS DE CIZALLA EN EL COMPLEJO QUEBRADAGRANDE EN LOS ALREDEDORES DE MANIZALES Y VILLAMARÍA
}

\author{
Mario Moreno-Sánchez ${ }^{1}$; Gustavo Hincapié J. ${ }^{1}$; César Augusto Ossa M. ${ }^{1}$; Luz Mary Toro Toro ${ }^{1}$
}

DOI: http://dx.doi.org/10.18273/revbol.v38n4-2016001 (c) (1)

Forma de citar: Moreno-Sánchez, M., Hincapié, G., Ossa, C.A., y Toro-Toro, L.M. 2016. Caracterización geológico-estructural de algunas zonas de cizalla en el Complejo Quebradagrande en los alrededores de Manizales y Villamaría. Boletín de Geología, 38(4): 15-27.

\section{RESUMEN}

En el Complejo Quebradagrande (en los alrededores de Manizales y Villamaría, Caldas) se identificaron tres zonas de rocas miloníticas de bajo grado que de oeste a este se denominaron: Los Túneles-Río Olivares, El Arroyo-Corinto y Gallinazo-Río Blanco. Los análisis micro- y macroscópicos permitieron identificar zonas de rocas miloníticas con una foliación que varía de N-S a NNE-SSW en rumbo, con buzamientos de intermedios a altos hacia el E, eventualmente con disposición subvertical. Indicadores cinemáticos tales como porfiroclastos tipo $\sigma$, estructuras S-C, estructuras S-C', permitieron determinar movimientos dextrales subhorizontales, dextrales-inversos hasta inversos, con varios ángulos de orientación de la lineación que muestran vergencia al SW. Se sugiere que el sistema de zonas de cizalla está relacionado con la acreción lateral de la Placa Caribe en el Cretácico Superior.

Palabras clave: zonas de cizalla, Complejo Quebradagrande, dextral

\section{GEOLOGICAL AND STRUCTURAL CHARACTERIZATION OF SOME SHEAR ZONES IN THE QUEBRADAGRANDE COMPLEX NEAR TO MANIZALES AND VILLAMARIA}

\begin{abstract}
The Quebradagrande Complex (Located in the vicinity of Manizales and Villamaria in the Department of Caldas, Colombia) exhibit three zones of low-grade mylonites. These were identified and named, from West to East as: Los Túneles-Río Olivares, El Arroyo-Corinto, and Gallinazo-Río Blanco. An analysis of the deformation mechanisms allow for the identification of areas where these mylonites show foliation with strikes ranging from $\mathrm{N} / \mathrm{S}$ to NE/SW, and moderate to high angle dips to the SE. In places dip angles are nearly vertical. Likewise, kinematic indicators such as type $\sigma$ porphyroclast, S-C structures and S-C' structures allowed to determine displacements right lateral, right lateral-reverse to reverse, with several vergence angles of the stretching lineation to the SW. It is suggested that the system of shear zones is related to the lateral accretion of the Caribbean plate in the Upper Cretaceous.
\end{abstract}

Keywords: shear zones, Quebradagrande Complex, dextral.

\footnotetext{
${ }^{1}$ Departamento de Ciencias Geológicas. Universidad de Caldas. Manizales, Colombia; (*) mario.moreno@ucaldas.edu.co, gustavo.hincapie@ucaldas.edu.co, ceossa@ucaldas.edu.co,luz.toro@ucaldas.edu.co
} 


\section{INTRODUCCIÓN}

El sector occidental de Colombia, al occidente de la Cordillera Central, está compuesto por una serie de bloques tectónicos con basamento de origen oceánico (Moreno-Sánchez and Pardo-Trujillo, 2003; Nivia et al., 2006; Restrepo and Toussaint, 1988). El bloque tectónico más oriental, denominado Complejo Quebradagrande (Maya y González, 1995), está constituido por fajas estrechas de rocas ígneas básicas y sedimentitas del Cretácico Superior delimitadas usualmente por zonas estrechas de cizalla cuya historia tectónica no está aún bien esclarecida.

El presente estudio se centra en el análisis de algunas zonas de cizallas dúctiles presentes en las rocas del Complejo Quebradagrande, las cuales dan lugar a rocas miloníticas desarrolladas a partir de un protolito de vulcanitas-tobas y sedimentitas. Dicho estudio fue realizado en los alrededores de Manizales, departamento de Caldas (FIGURA 1). La interpretación cinemática de las rocas deformadas es una fuente directa de información para la reconstrucción de la evolución tectónica de la zona. Es de anotar que en el área es posible hallar franjas de rocas sedimentarias y volcano-sedimentarias pertenecientes al Complejo Quebradagrande en las que no hay deformación milonítica, tales franjas muestran una serie de fallas inversas. Este estudio se centra en las zonas de cizalla con desarrollos de rocas miloníticas. Estas zonas se encuentran, a menudo, cubiertas por una espesa capa de suelos de origen vegetal y material piroclástico (lapilli y ceniza).

\section{MARCO GEOLÓGICO}

Localizada en el noroeste de Colombia, la Cordillera Central está constituida por bloques tectónicos de naturaleza oceánica y continental, entre los cuales destacan el Complejo Quebradagrande (Maya y González, 1995). Grosso modo, el complejo es una entidad tectoestratigráfica con extensión regional conformada por bloques estructurales con tendencias $\mathrm{N}-\mathrm{S}$ constituido por dos litodemas que se diferencian con base en sus rasgos petrográficos y geoquímicos (Moreno-Sánchez et al., 2008). La edad del complejo, basada en fósiles, abarca el lapso Berriasiano-Albiano (Moreno-Sánchez and Pardo-Trujillo, 2003), sin embargo, edades $\mathrm{U} / \mathrm{Pb}$ en circones sugieren que la sedimentación alcanza el Cenomaniano (Tamayo y Correa, 2010). Las rocas ígneas en el complejo presentan una naturaleza mixta, con asociaciones de gabros y basaltos de procedencia oceánica (Toro-Toro et al., 2010) superpuestos por andesitas y basaltos calcoalcalinos (Nivia et al., 2006) formados en un arco volcánico. Existe cierto consenso en que el origen del Complejo Quebradagrande está relacionado con el desarrollo en el occidente colombiano de una cuenca marginal (backarc basin), ligada a un arco volcánico que se expandió hasta producir un fondo oceánico (Álvarez, 1983; Moreno-Sánchez and PardoTrujillo, 2003). Según Nivia et al. (2006), el Complejo Quebradagrande se formó al comienzo del Cretácico como una cuenca marginal ensiálica que se cerró durante la acreción de bloques de corteza oceánica afines con los basaltos de meseta oceánica de la Placa Caribe (Caribbean-Colombian Cretaceous oceanic plateau).

Los límites estructurales del Complejo Quebradagrande corresponden en el sector oriental con la falla San Jerónimo y en el sector occidental con la falla SilviaPijao (Maya y González, 1995). González (1977) reconoce la existencia de zonas miloníticas en el Complejo Quebradagrande pero sin profundizar en su significado cinemático. Nivia et al. (2006) advierte que las rocas miloníticas en el complejo a menudo han sido tratadas como rocas producto de un metamorfismo orogénico.

\section{METODOLOGÍA}

La metodología empleada para el desarrollo de este trabajo incluye la recopilación, revisión y análisis de la literatura disponible que se considera más pertinente para este trabajo relacionada con zonas de cizalla (Arancibia et al., 1999; Massey and Moecher, 2013; Passchier and Trouw, 2005; Sibson, 1977; Trouw et al., 2010). Se seleccionaron las quebradas que representan cinco transectos: río Blanco, quebrada El Águila y quebrada La Francia en el sector norte; y las quebradas El Arroyo y Gallinazo en el sector sur. Las estructuras en el área de estudio (coordenadas $\mathrm{N}=1$ '062.000, $\mathrm{S}=1$ '044.000, $\mathrm{W}=835.000, \mathrm{E}=853.000$, con origen Bogotá, plancha 206 del IGAC, FIGURA 1) se levantaron a una escala de 1:25.000. Para cada muestra de roca se realizó un corte perpendicular a la foliación milonítica y paralela a la dirección de estiramiento de los minerales. Desde el punto de vista microscópico las rocas estudiadas muestran una variedad de estructuras tales como: textura milonítica, orientación preferencial de agregados de minerales, porfiroclastos de tipo $\sigma \mathrm{y} \delta$, peces de mica y estructuras S-C y S- C'. Los diagramas equiareales de contornos se elaboraron con el programa Open Stereo (Grohmann and Campanha, 2010). 


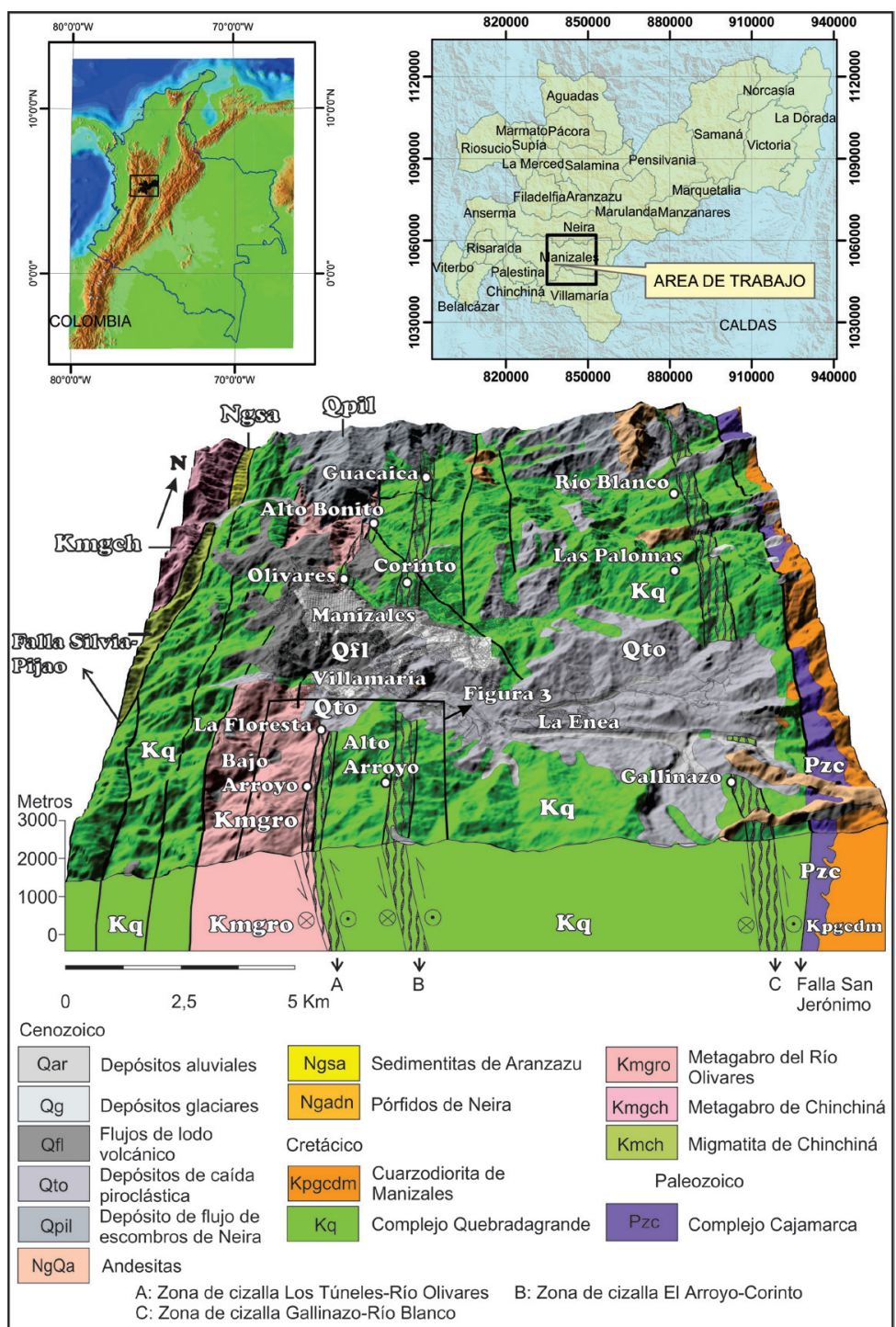

FIGURA 1. Localización del área de estudio y bloque diagrama que muestra las zonas de cizalla analizadas. Geología modificada de Mosquera et al. (1998).

\section{ZONAS DE CIZALLA \\ DESARROLLADAS EN EL COMPLEJO QUEBRADAGRANDE}

De acuerdo con los resultados de los análisis meso y microscópicos, las rocas del Complejo Quebradagrande fueron deformadas, al menos en tres zonas, en las que la deformación ocurrió inicialmente en el nivel dúctil con sobreimposición de deformación en el nivel frágil. La deformación dúctil se manifiesta por el desarrollo de rocas milonitas y la deformación frágil por rocas cataclásticas. En algunas zonas de cizalla se generaron venas de pseudotaquilitas.

De oeste a este, las denominadas zonas de cizalla son: Los Túneles-río Olivares, El Arroyo-Corinto y
Gallinazo-Río Blanco (FIGURA 1). Las zonas de cizalla se orientan N-S a NNE-SSW, con variaciones a NNWSSE. El buzamiento dominante en las zonas de cizalla es alto, inclinándose ligeramente al E, pero llegando en algunos casos a ser subvertical. Debido a sus límites difusos no se pudo determinar el ancho preciso de las zonas de cizalla, aunque su espesor es del orden de varias decenas de metros. Cada zona está caracterizada meso y microscópicamente por dominios anastomosados de rocas miloníticas que separan bloques lenticulares con aparente menor deformación por cizalla dúctil. Las zonas de cizalla ocasionalmente se encuentran cubiertas por depósitos sedimentarios de edad Pleistocena (Formaciones Manizales y Casabianca), por rocas volcánicas piroclásticas y por la infraestructura urbana de los municipios de Manizales y Villamaría. 
En las franjas separadas por las zonas de cizalla dúctil, las rocas del Complejo Quebradagrande se pueden ver sin deformación milonítica, con rocas sedimentarias con estructuras de estratificación plana y ondulada (FIGURA
2A). También es posible encontrar moldes de amonitas, de edad Albiano (FIGURA 2B). La deformación hallada en estas franjas corresponde a una serie de fallas inversas con buzamientos hacia el SE y vergencia hacia el SW.
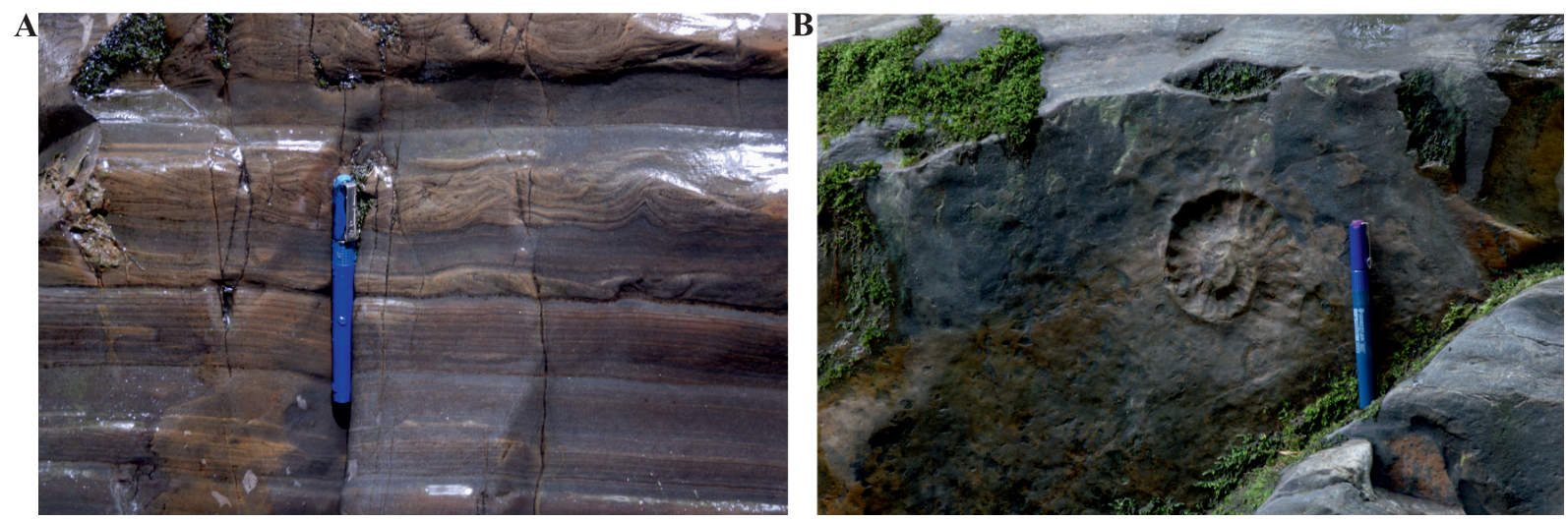

FIGURA 2. A. Rocas sedimentarias indeformadas de origen turbidítico (estratificación plana y ondulada) pertenecientes al Complejo Quebradagrande, vereda Arroyo Alto, Villamaría; B. Impresión de amonita de edad Albiano, vereda Arroyo Alto, Villamaría.

\section{ZONA DE CIZALLA LOS TÚNELES- RÍO OLIVARES}

Esta zona de cizalla está ubicada hacia la parte oeste del área de estudio, a unos $5 \mathrm{~km}$ de la Falla SilviaPijao, la cual constituye el límite occidental del Complejo Quebradagrande. Esta zona de cizalla tiene una orientación N-S, pone en contacto las rocas del Metagabro del Río Olivares (Hincapié et al., 2010) al oeste, con las rocas volcano-sedimentarias (tobas) deformadas del Complejo Quebradagrande al este. Su traza se puede seguir de sur a norte por las veredas Bajo Arroyo, Floresta, Olivares y Alto Bonito. La zona de milonitas asociada con esta zona de cizalla coincide con el trazo de la Falla Manizales-Aranzazu (Mosquera et al., 1998). Los mejores afloramientos se encuentran sobre la antigua vía del ferrocarril (sector de los túneles) y cerca al cauce del río Olivares, en la parte norte de la zona.

Las rocas de la zona de cizalla presentan una foliación milonítica muy bien desarrollada, en la que la lineación de minerales es difícil reconocer a escala de afloramiento, debido al tamaño de grano muy fino de los minerales; no obstante, en la FIGURA 3 se muestra la porción suroeste de la zona de estudio en la que se ubican algunos datos estructurales para la foliación milonítica y la lineación de minerales. La foliación tiene tendencia N-S a NNESSW, con buzamientos altos al E y SE a subverticales (FIGURA 4). A escala mesoscópica la deformación se caracteriza por zonas de milonitización de escala métrica que separan bloques de roca menos afectados por la deformación.

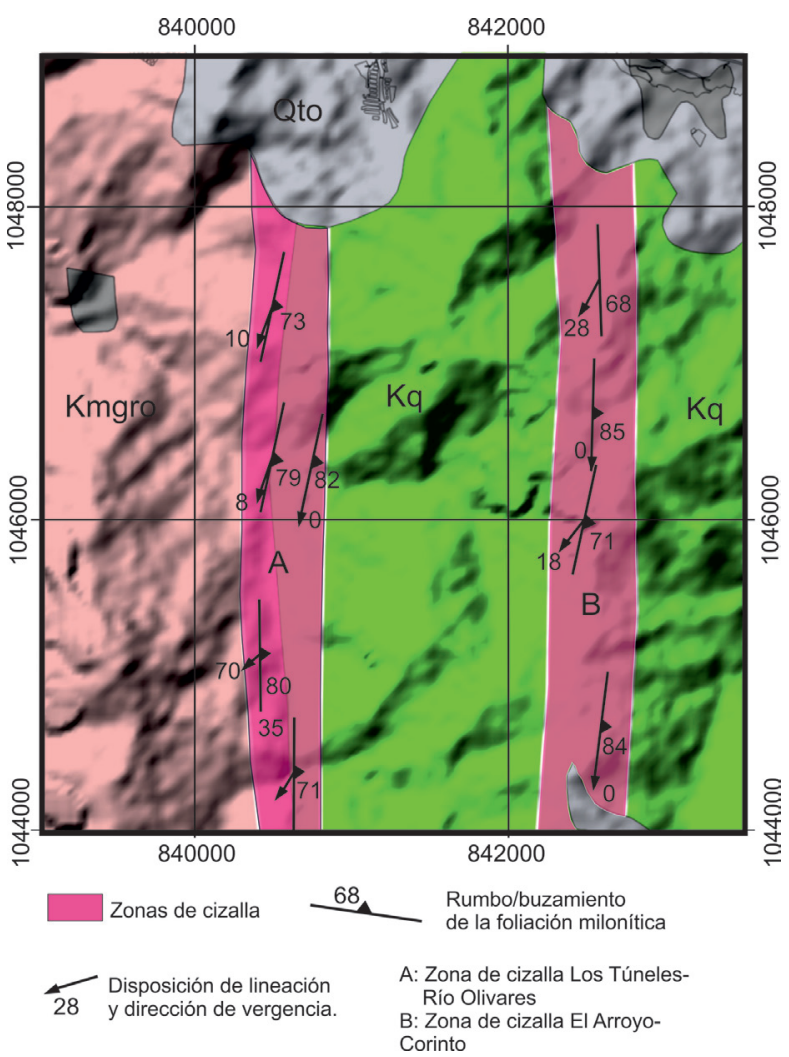

FIGURA 3. Detalle de la zona suroeste del área de estudio. Se observan las zonas de cizalla Los túneles-río Olivares y El Arroyo-Corinto, con algunos datos estructurales de la disposición de la foliación milonítica y vergencia obtenida a partir de lineaciones. El espesor de las zonas de cizalla es sólo representativo, los límites de las zonas no son netos. La cubierta vegetal no permite determinar los espesores de modo preciso. Códigos de las unidades como en la FIGURA 1. 


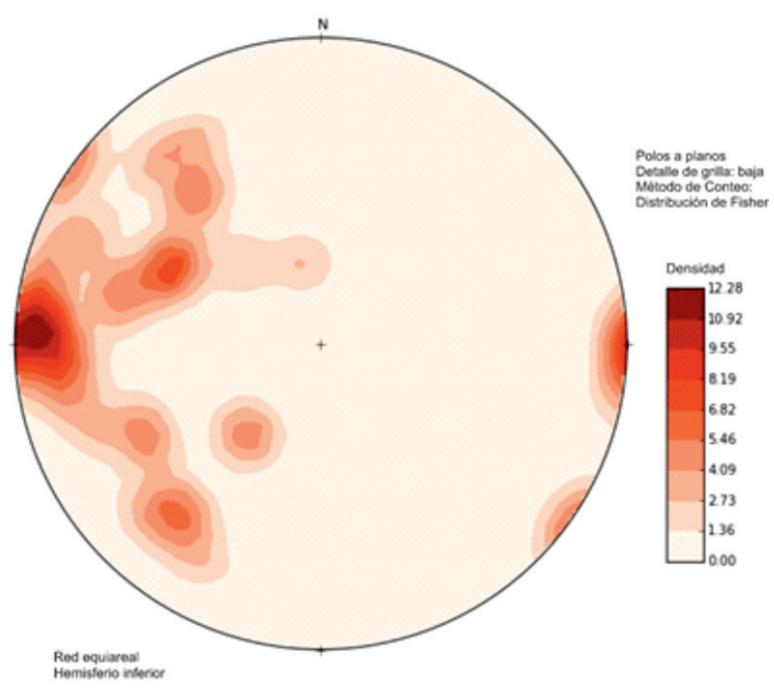

FIGURA 4. Diagrama equiareal de contornos para polos de planos de la foliación milonítica, tomados en afloramientos correspondientes a la zona de cizalla Los Túneles-río Olivares. La foliación tiene tendencia N-S a NNE-SSW, con buzamientos de altos al SE a subverticales. Proyección en el hemisferio inferior. Número de datos: 34 . Diagrama elaborado con Open Stereo (Grohmann and Campanha, 2010).

Los protolitos de las milonitas cuarzo-feldespáticas corresponden a tobas lítico-cristalinas. Las milonitas están compuestas por porfiroclastos de rocas sedimentarias (lodolitas) y volcánicas (basaltos y andesitas), rodeados por una matriz microcristalina conformada principalmente por filosilicatos, agregados muy finos de cuarzo-feldespato y óxidos de hierrotitanio. La foliación milonítica es penetrativa. Los indicadores cinemáticos hallados son porfiroclastos de fragmentos de cristales de plagioclasa y de líticos (andesitas-lodolitas) con colas tipo $\sigma$ (FIGURA 5A), sombras de presión asimétrica compuestas por material micáceo de tamaño muy fino (Passchier and Trouw, 2005), y bandas de cizalla con estructuras de tipo S-C (FIGURA 5) y S-C'.

En sección delgada, los indicadores cinemáticos establecen un sentido de cizallamiento general dextral (FIGURA 5). Al orientar las secciones delgadas según su posición de campo resulta una cinemática dextral subhorizontal con variaciones a dextral inversa, consistente con la cinemática determinada con otros indicadores mesoscópicos en algunos afloramientos. El análisis de los mecanismos y microestructuras de deformación en las milonitas tobáceas permiten clasificarlas como milonitas de bajo grado, según la clasificación de Trouw et al. (2010), que establecen temperaturas de $250^{\circ}$ a $350^{\circ} \mathrm{C}$ durante el desarrollo de la deformación. El cuarzo presenta subgranos (FIGURA $5 \mathrm{E})$ y extinción ondulatoria de barrido, las plagioclasas tienen fracturamiento y una incipiente extinción ondulatoria de barrido.

En el Metagabro del Río Olivares (Hincapié et al., 2010), el cual forma una faja continúa hacia el sur hasta el sector de Los Túneles, se presenta una serie de zonas de rocas milonitizadas, con espesores centimétricos, cuya tendencia estructural va desde N-S hasta NNE-SSW, con buzamientos altos al SE llegando a ser subverticales (FIGURA 4). Las rocas deformadas corresponden a protomilonitas y milonitas, las cuales tienen porfiroclastos de plagioclasa y piroxenos dentro de una constituida por minerales neoformados (clorita, actinolita y epidota).

En las plagioclasas se observan cristales maclados mecánicamente y flexionados, extinción ondulatoria de barrido y estructuras núcleo-manto. En los clinopiroxenos se presenta extinción a parches y extinción ondulatoria de barrido. Estos mecanismos de deformación indican una temperatura de deformación más alta para la franja de metagabros, en relación a las milonitas tobáceas, lo que sugiere que éstos estuvieron a un nivel cortical más profundo. La cinemática hallada en sección delgada y afloramiento es similar a la de las milonitas tobáceas.

El clinopiroxeno aparece como mineral relicto (augita $25 \%$ modal) totalmente rodeado por anfíbol fibroso, con tamaños de $0,4 \mathrm{~mm}$ y con inclusiones de epidota y opacos. Las plagioclasas ( $45 \%$ modal) son subhedrales y presentan inclusiones de clorita y epidota. Es muy frecuente observar inclusiones de apatito acicular en las plagioclasas. Algunas plagioclasas están fuertemente alteradas a una masa oscura (sausuritización). Los minerales neoformados son clorita, epidota, cuarzo y actinolita, estos últimos minerales alcanzan un porcentaje en el rango 10-30 $\%$ de la roca total.

Las rocas miloníticas tienen sobreimpuesta una deformación frágil marcada por la presencia de minerales rotos por cataclasis, fracturamiento inter e intragranular y por zonas de cataclasis.

En el sector de los Túneles se encontraron venas de pseudotaquilitas, asociadas con el metagabro. Estas pseutotaquilitas se caracterizan por presentar fragmentos angulares del metagabro con tamaños menores a $0,3 \mathrm{~mm}$ (FIGURA 5F). En los metagabros que afloran en el sector norte del área del río Olivares, Hincapié et al. (2010) describe la presencia de pseudotaquilitas. 

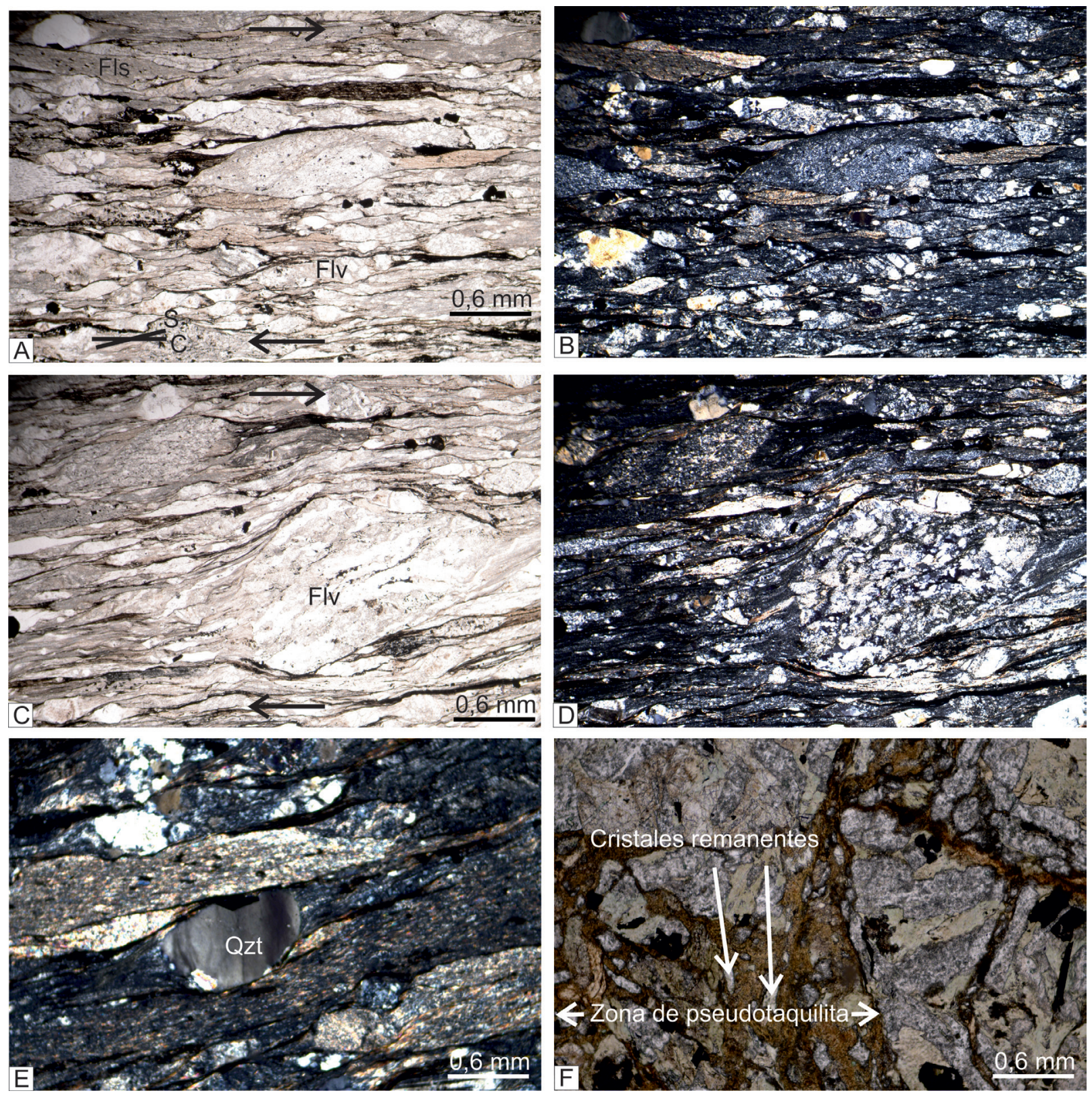

FIGURA 5. A-B. Porfiroclastos de fragmentos líticos de rocas volcánicas (Flv) y rocas sedimentarias (lodolitas, Fls). Los porfiroclastos son tipo $\sigma$, indican un sentido de cizalla dextral. A. Nicoles paralelos en las milonitas, B. Nicoles cruzados. C-D. Porfiroclasto tipo $\sigma$, de fragmento lítico de roca volcánica (Flv), sentido de cizalla dextral. A. Nicoles paralelos, B. Nicoles cruzados. E. Porfiroclasto de cuarzo (Qzt) con desarrollo de subgranos y extinción ondulatoria de barrido. Los subgranos se disponen en forma vertical en la fotografía. Nicoles cruzados. F. Detalle de zona de pseudotaquilita y cataclasis en el metagabro. Nicoles paralelos. Cortes perpendiculares a la foliación milonítica y paralelos a la lineación de alargamiento de minerales. Rocas de la zona de cizalla Los túneles-Río Olivares.

\section{ZONA DE CIZALLA EL ARROYO- CORINTO}

Esta zona de cizalla se encuentra al este de la zona anterior; se extiende por las veredas Alto Arroyo, Tejares, Sierra Morena y Guacaica (FIGURA 1), presenta un espesor estructural de aproximadamente $800 \mathrm{~m}$, una tendencia de N-S a NNE-SSW y con buzamientos altos a intermedios hacia el E. La foliación milonítica se dispone preferencialmente al NNE-SSW, con buzamientos de intermedios a altos al E (FIGURA 6). La disposición de la lineación mineral varía de subhorizontal hasta 
ángulos de $70^{\circ}$, con vergencia al SW (FIGURA 3), eventualmente incluso de $90^{\circ}$. Regionalmente, la zona de cizalla está compuesta internamente por varias zonas de deformación anastomosadas, con desarrollo de milonitas a partir de tobas y rocas sedimentarias (lodolitas). A escala de afloramiento muestra dominios con foliación anastomosada separando dominios de roca poco o nada cizallados, a microescala, se observan zonas de ultramilonitas de espesores milimétricos a centimétricos, dentro de milonitas y protomilonitas (FIGURA 7A).

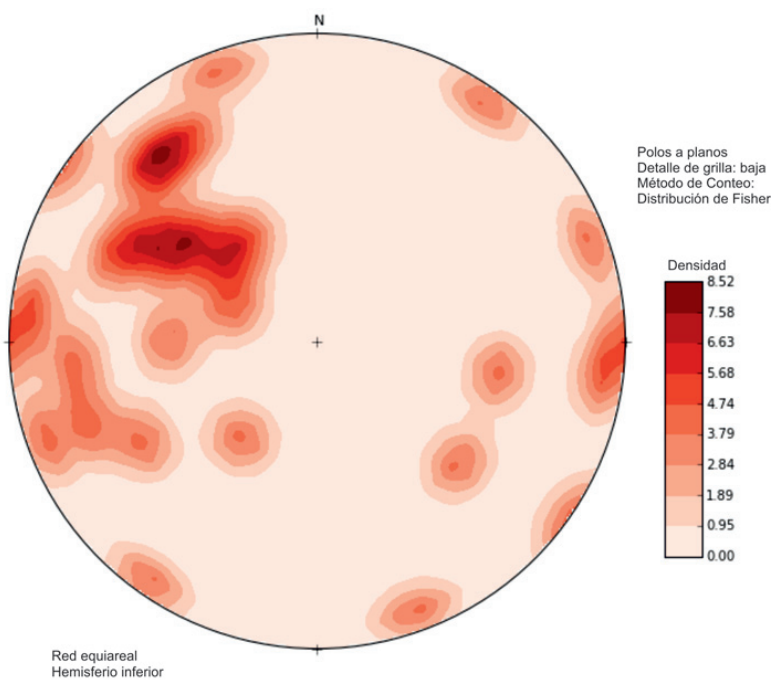

FIGURA 6. Diagrama equiareal de contornos para polos de planos de la foliación milonítica, tomados en la zona de cizalla El Arroyo-Corinto. La foliación milonítica se dispone preferencialmente al NNE-SSW, con buzamientos de intermedios a altos al este. Proyección en el hemisferio inferior. Número de datos: 23. Diagrama elaborado con Open Stereo (Grohmann and Campanha, 2010).

Esta zona de cizalla constituye el límite entre el sector oriental del Complejo Quebradagrande, donde predominan rocas sedimentarias, y el sector occidental del mismo complejo donde hay mayor cantidad de rocas volcánicas. Los sitios donde mejor se observa la zona de cizalla son: parte media de la quebrada El Arroyo, sectores urbanos de Manizales, vereda Corinto (quebrada Caracola) y río Guacaica.

Las rocas volcanoclásticas y sedimentarias afectadas por milonitización son tobas lítico-cristalina con vidrio, margas y cuarzo-arenitas principalmente. En general, todas las rocas que se describen en este trabajo presentan una foliación. Para el caso de las milonitas tobáceas (FIGURA 7B), los porfiroclastos están compuestos por fragmentos líticos de rocas sedimentarias (lodolitas) y fragmentos de rocas volcánicas (basaltos). Eventualmente se presenta cuarzo recristalizado a un agregado de subgranos elongados y líticos de rocas sedimentarias y volcánicas, con tamaños entre 0,5 y $0,9 \mathrm{~mm}$. La matriz está compuesta por bandas de filosilicatos (FIGURA 7B). Las milonitas cuarzo-feldespáticas, derivadas de cuarzo-arenitas y wackas arcósicas, presentan porfiroclastos de cuarzo y plagioclasa. La matriz está definida por una alternancia milimétrica de bandas cuarzo-feldespáticas y bandas de moscovita y grafito de tamaño de grano muy fino. Según la proporción porfiroclastos-matriz, las rocas varían de protomilonitas a ultramilonitas, pasando por milonitas (Sibson, 1977).

La cinemática se determinó mediante la utilización de estructuras S-C (FIGURA 7C), porfiroclastos tipo $\sigma$ (FIGURAS 7C y 7D), sombras de deformación de filosilicatos (FIGURA 7D) y porfiroclastos con fracturas sintéticas. El sentido de cizallamiento resultante fue dextral en secciones delgadas, a nivel de restitución de campo los movimientos varían de dextrales a dextrales inversos.

Los mecanismos microscópicos de deformación en cuarzo corresponden a extinción ondulatoria de barrido, recristalización dinámica por migración de borde de grano (FIGURA 7E) y en ocasiones por rotación de subgranos. La plagioclasa presenta maclamiento mecánico, fracturamiento y esporádicamente extinción ondulatoria de barrido. La moscovita está deformada por flexión y por extinción ondulatoria de barrido (FIGURA 7F). Estos mecanismos permiten clasificar estas rocas como milonitas de bajo grado (Trouw et al., 2010) con temperaturas de deformación entre 250 y 350 ${ }^{\circ} \mathrm{C}$. La edad de esta zona de cizalla debe ser posterior a la edad de las sedimentitas afectadas que según datos geocronológicos $(\mathrm{U} / \mathrm{Pb}$ en circones detríticos) obtenidos de la sección del río Olivares aportan una edad máxima del Cenomaniano (Tamayo y Correa, 2010).

\section{ZONA DE CIZALLA GALLINAZO-RÍO CLARO}

Esta es la zona de cizalla más oriental de la zona estudiada y se ubica cerca de la Falla San Jerónimo, cruzando las veredas de Gallinazo, Las Palomas y Río Blanco, entre otras (FIGURA 1). En su conjunto presenta una dirección de N-S a NNE-SSW y ángulos de buzamientos de intermedios a altos hacia el $\mathrm{E}$ (FIGURA 8). La lineación de alargamiento de minerales tiene disposiciones de subhorizontales a subverticales con disposiciones que permiten deducir vergencia al SW, lo que sugiere que la zona de deformación pudo haber tenido componente de cizalla simple, que genera 
transcurrencia dextral y componente de cizalla pura, la cual generó acortamiento. No fue posible determinar su espesor, pero es observable por decenas de metros. Esta zona se caracteriza por la presencia de zonas miloniticas anastomosadas, rodeando bloques lenticulares de roca poco cizallados. La cinemática en la zona de cizalla varía de dextral a dextral inversa, con algunos movimientos inversos puros, lo cual se deduce de los datos hallados en afloramiento y en sección delgada.
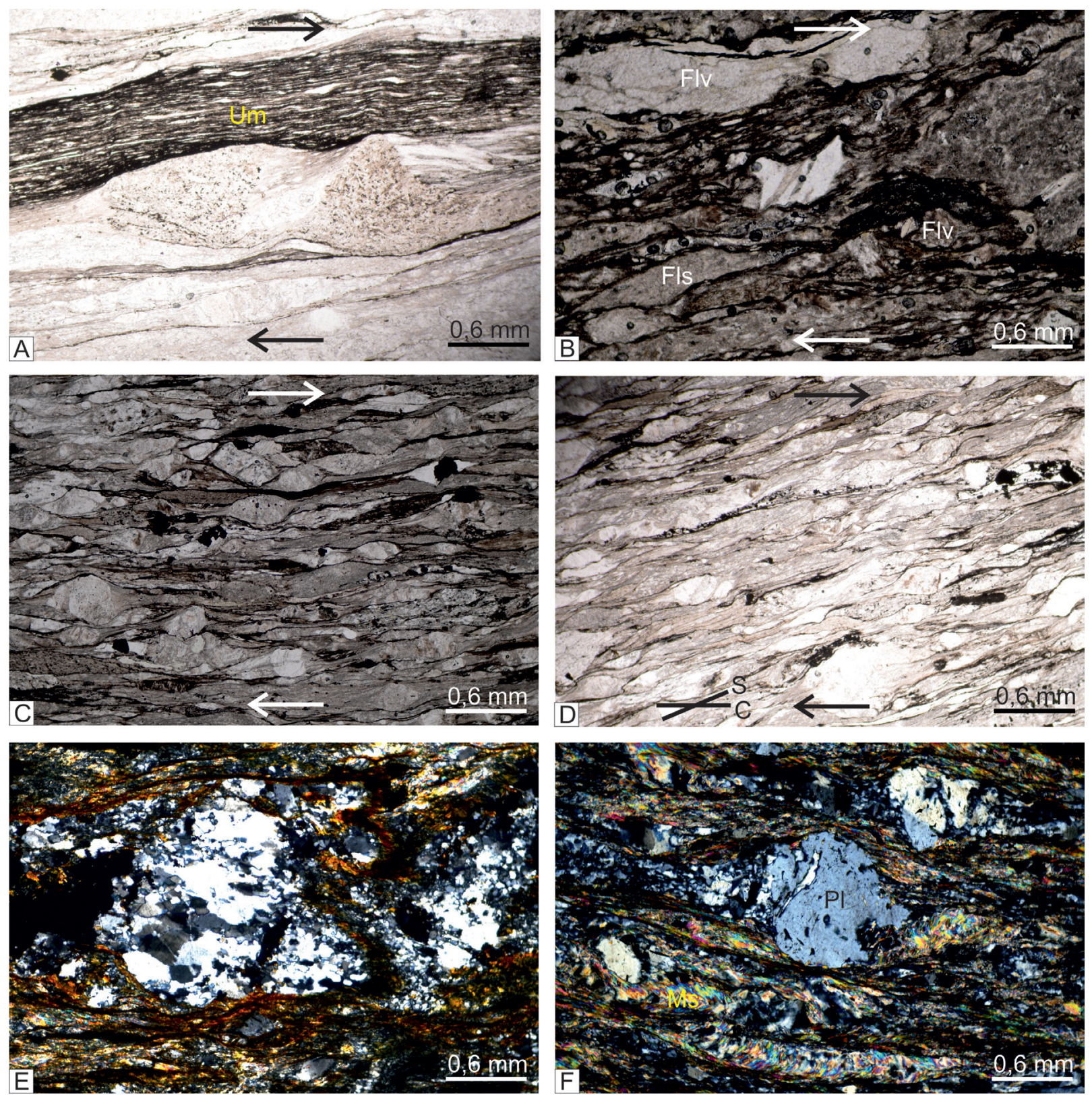

FIGURA 7. A. Milonita a partir de tobas con niveles de ultramilonitas (Um). Sentido de cizalla dextral. Nicoles paralelos. B. Milonita con porfiroclastos de fragmentos líticos de rocas volcánicas (Flv) y rocas sedimentarias (Fls). Nicoles paralelos. C. Sombras de deformación en porfiroclastos tipo $\sigma$, sentido de cizalla dextral. Nicoles paralelos. D. Milonita constituida por porfiroclastos tipo $\sigma$, con desarrollo de estructura $\mathrm{S}-\mathrm{C}$, la cual indica cizalla dextral. Nicoles paralelos E. Porfiroclasto de cuarzo con recristalización dinámica por migración de borde de grano. Nicoles cruzados. F. Láminas de moscovita flexionada (Ms) y con extinción ondulatoria de barrido. Nicoles cruzados. Porfiroclasto de plagioclasa (Pl) tipo $\delta$. El corte de las secciones delgadas es perpendicular a la foliación milonítica y paralelo a la lineación de estiramiento. Rocas de la zona de cizalla El Arroyo-Corinto. 
Los mejores afloramientos se hallan por el río Chinchiná, cerca de la planta del acueducto Luis Prieto y por la carretera que conduce hacia la reserva de Río Blanco. Las milonitas que se desarrollaron en la zona de cizalla se derivan de cuarzoarenitas, wackas arcósicas y margas principalmente. Las milonitas cuarzo-feldespáticas se componen esencialmente de porfiroclastos de cuarzo y plagioclasa, cuyos tamaños varían entre 0,5 y $0,7 \mathrm{~mm}$, en una matriz dúctil de cuarzo, materia orgánica, clorita y óxidos de hierro. Las milonitas derivadas de margas están compuestas por porfiroclastos de cuarzo y plagioclasas en una matriz definida por bandas de carbonatos, filosilicatos y cuarzo recristalizado.

Los indicadores cinemáticos utilizados son bandas de cuarzo asimétricas, porfiroclastos, tipo sigma (FIGURA 9A), peces de mica y estructuras S-C (FIGURA 9A). En sección delgada la cinemática es dextral, al restituir las secciones a su posición de campo la cinemática corresponde a dextral, dextral inversa y ocasionalmente inversa.

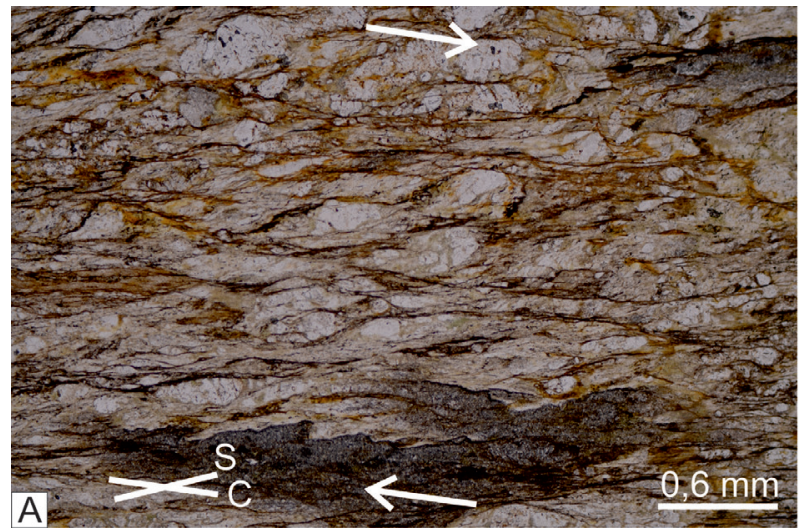

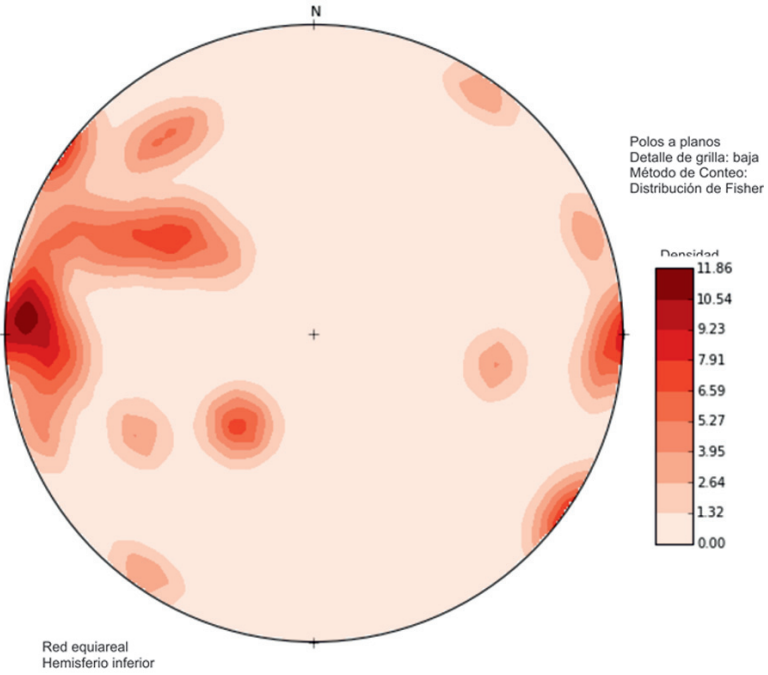

FIGURA 8. Diagrama equiareal de contornos para polos de planos de la foliación milonítica, tomados en la zona de cizalla Gallinazo-río Blanco. Los datos tienen una tendencia N-S a NNE-SSW, con buzamientos de intermedios a altos hacia el SE. Proyección en el hemisferio inferior. Número de datos: 24. Diagrama elaborado con Open Stereo (Grohmann and Campanha, 2010).

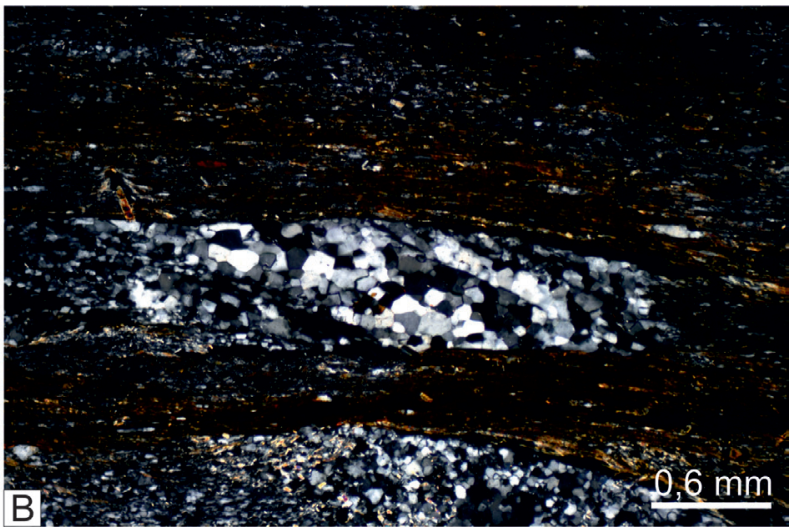

FIGURA 9. A. Estructuras S-C, en milonitas cuarzo-feldespáticas, las cuales indican una cinemática dextral. Nicoles paralelos. B. Cuarzo con recristalización estática, los nuevos granos tienen contactos poligonales. El corte de las secciones delgadas es perpendicular a la foliación milonítica y paralelo a la lineación de estiramiento. Rocas de la zona de cizalla Gallinazo-Río Blanco.

Las rocas se clasificaron como de bajo a medio grado (Trouw et al., 2010), correspondiente a temperaturas estimadas entre 250 y $350{ }^{\circ} \mathrm{C}$. Los mecanismos microscópicos hallados en cuarzo son: extinción ondulatoria de barrido, recristalización por rotación de subgranos. Algunos granos presentan recristalización evidenciada por la presencia de contactos poligonales (FIGURA 9B).

La zona de cizalla está cortada por una serie de fallas transcurrentes dextrales, (FIGURA 10). Localmente, en la vía hacia la reserva Río Blanco, la zona de cizalla es afectada por una faja de deformación frágil en la que se desarrollaron una serie de fallas inversas con disposición E-W y con buzamientos bajos (alrededor de $30^{\circ}$ ) al sur. El movimiento del bloque de falla genera un replegamiento de la foliación milonítica, donde se desarrollan pliegues intrafoliales y un pliegue por flexión de falla, los cuales indican vergencia hacia el oeste (FIGURA 11).

La presencia de moluscos fósiles (Gómez-Cruz et al., 1995) permiten deducir que la edad de sedimentación de las rocas en esta área es Albiense lo que establece una edad posterior para la deformación. 


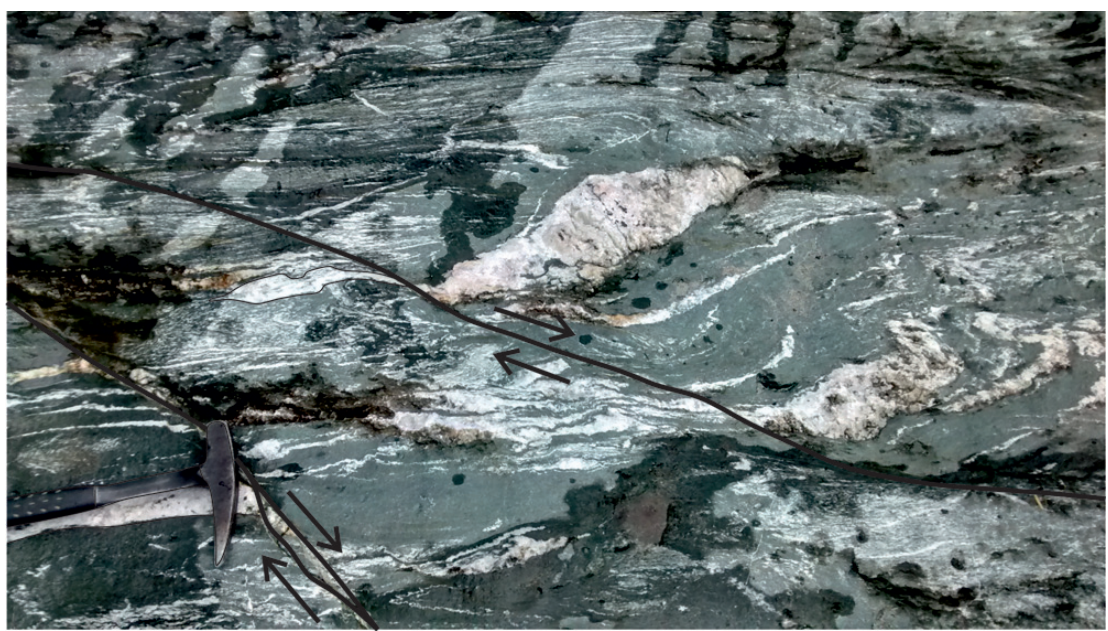

FIGURA 10. Fallas transcurrentes dextrales, las cuales cortan a las milonitas de la zona de cizalla Gallinazo-río Blanco. Vista de planta. Río Chinchiná, cerca de la planta del acueducto Luis Prieto.
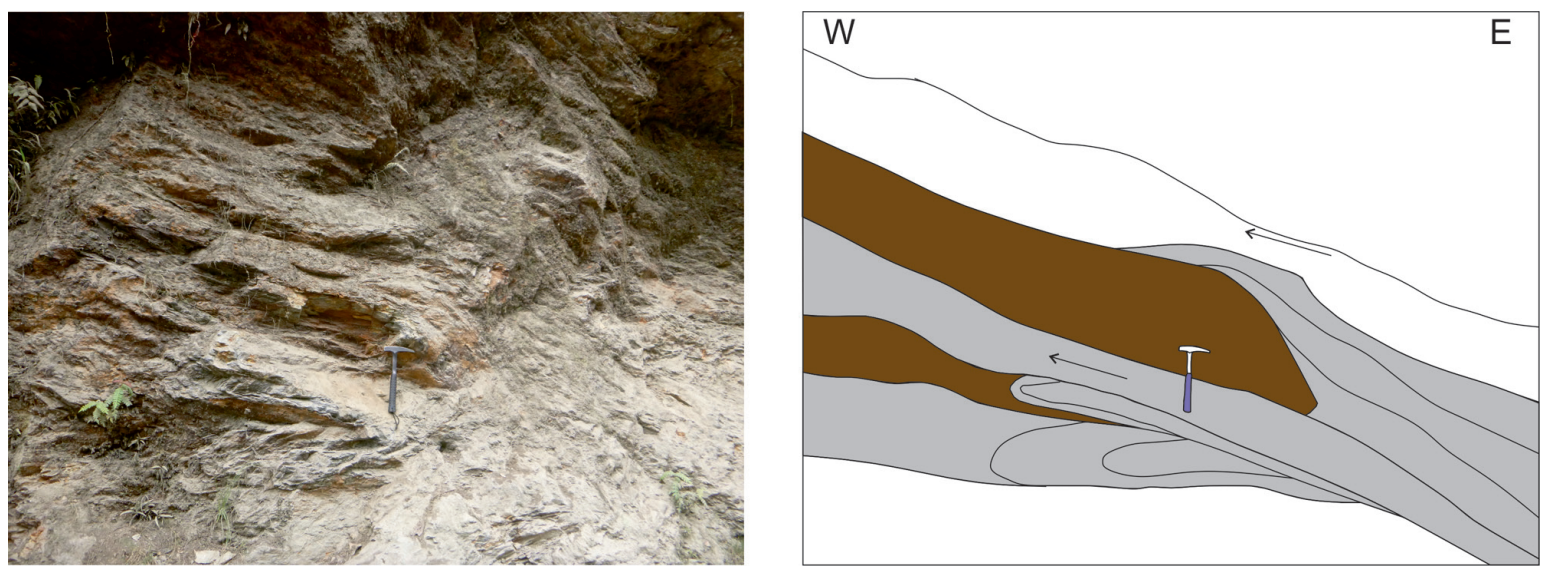

FIGURA 11. Fallas inversas, las cuales forman replegamientos en las milonitas, los pliegues permiten deducir una vergencia hacia el oeste. Carretera La Sultana-reserva Río Blanco, cerca de la planta del acueducto

\section{DISCUSIÓN}

Las observaciones realizadas en campo como a nivel microscópico en las rocas deformadas del Complejo Quebradagrande indican la existencia de un sistema de zonas de cizalla que desarrollaron rocas de protomilonitas hasta milonitas y ultramilonitas. Las tres zonas de cizalla analizadas en este trabajo presentan condiciones cinemáticas y deformativas similares. Todas ellas se caracterizan, a escala mesoscópica, por el desarrollo de zonas miloníticas de geometría anastomosadas a subparalelas que rodean e individualizan bloques de rocas poco o nada deformadas por el cizallamiento. A escala microscópica las milonitas analizadas presentan el desarrollo de bandas de ultramilonitas dentro de protomilonitas y milonitas definidas por diferentes relaciones de porfiroclastos y matriz. También se reportan bandas ricas en filosilicatos propicias para acomodar deslizamiento.
Las orientaciones de las zonas de cizalla son muy similares, con rumbos que varían entre N-S y NNESSW y buzamientos de intermedios a altos hacia el E y SE, los cuales pueden llegar a ser subverticales. Los indicadores cinemáticos analizados a escalas mesoscópica y microscópica permiten deducir movimientos dextrales, dextrales inversos e inversos. Las lineaciones de alargamiento de minerales tienen disposiciones que varían entre subhorizontales a subverticales, pasando por oblicuos con vergencia al SW. Estas combinaciones de movimientos se pueden desarrollar en zonas influenciadas por transpresión y consecuente partición de la deformación (estos términos se utilizan en el sentido de Fossen, 2010).

Teniendo en cuenta las ideas propuestas para transpresión y partición de la deformación, los resultados del análisis a escala meso y microscópica de las zonas de cizalla, indican que la deformación de las rocas del 
Complejo Quebradagrande tuvo lugar en un régimen de transpresión dextral, el cual se caracteriza por una componente subhorizontal de cizalla simple y una componente subhorizontal ortogonal de cizalla pura, que produce aplastamiento y elongación subvertical.

De acuerdo con los mecanismos microscópicos de la deformación se puede postular que las rocas de la serie de las milonitas desarrolladas en las zonas de cizalla corresponden a milonitas de bajo grado, y en el caso de la zona de cizalla Gallinazo-Río Claro las milonitas son de facies Esquisto Verde de baja temperatura.

El Complejo Quebradagrande se origina a partir de una cuenca marginal (back arc basin) con un basamento de espesor desigual que podría condicionar la deformación posterior. Se sugiere que las zonas de mayor deformación estarían situadas preferencialmente sobre zonas de basamento adelgazado. Las áreas con menor deformación estarían sobre sectores engrosados o rígidos de basamento.

\section{RELACIÓN ENTRE TRANSPRESIÓN Y MODELOS EVOLUTIVOS PARA COLOMBIA}

El primer trabajo que establece para el occidente de Colombia un modelo de evolución histórica ajustado a la nueva teoría de tectónica de placas fue el de Barrero (1979). De acuerdo con este autor, durante el Cretácico, las acreciones de bloques al occidente de la Cordillera Central se explicarían por subducción más o menos ortogonal contra el margen sudamericano. Sin embargo, los nuevos datos geológicos para el occidente colombiano condujeron el desarrollo de modelos en donde la transpresión juega un papel importante durante la acreción de terrenos. Giunta et al. (2002) proponen un modelo de evolución geológica donde la placa Caribe se origina en su posición actual (in situ), sin embargo, esta hipótesis falla al no explicar la complejidad estructural y geoquímica del Occidente Colombiano. En el modelo de Pindell (1993; 1994; 1997), Pindell et al. (1998) y Pindell and Kennan (2009) la placa Caribe se origina en el océano pacifico y se desplaza hacia el NE colisionando lateralmente con el margen occidental colombiana durante el Cretácico Tardío. Según MorenoSánchez and Pardo-Trujillo (2003) el Complejo Quebradagrande representa una cuenca ensiálica (backarc basin) formada en el margen occidental de Colombia y Ecuador durante el Cretácico Temprano y que constituye una prolongación al sur del Protocaribe (Proto-Caribbean Sea Way) de Pindell (1993).
Durante el Cretácico Tardío se cierra la cuenca marginal del Quebradagrande como resultado de la colisión diagonal de la Placa Caribe. En este trabajo se sugiere que es en esta fase cuando se produce la ruptura y el desplazamiento dextral de los bloques que forman el Complejo Quebradagrande y los terrenos con basamento continental de la Cordillera Central. Martens et al. (2014) teoriza que los terrenos paleozoicos que constituyen parcialmente la Cordillera Central se originaron más al sur (Perú) de su posición actual y registran eventos tectónicos compartidos con los terrenos del sur de México amalgamados al margen occidental de Gondwana durante el Paleozoico.

El Complejo Quebradagrande presenta rocas que comprenden un rango que abarca el Berriasiano hasta el Albiano (Moreno-Sánchez et al., 2008) con probable interrupción de la sedimentación en el Cenomaniano. La presencia de fósiles del Albiano (Gómez-Cruz et al., 1995) y circones detríticos que alcanzan el Cenomaniano (Tamayo y Correa, 2010) en las rocas afectadas por cizalla sugieren que los eventos tectónicos que las produjeron datan al menos del Cretácico Tardío. Se sugiere además que la edad de deformación podría ser similar a la obtenida por Vinasco and Cordani (2012) en micas neoformadas y zonas miloníticas de la Falla de San Jerónimo que la sitúan entre 87-90 Ma y 72-81 Ma (método ${ }^{39} \mathrm{Ar}-{ }^{40} \mathrm{Ar}$ ).

\section{CONCLUSIONES}

Los análisis mesoscópicos y microscópicos en las rocas del Complejo Quebradagrande, permitieron definir al menos tres zonas de cizalla en las que se desarrollaron protomilonitas, milonitas y ultramilonitas. Las zonas de cizalla se denominaron informalmente Los túneles-río Olivares, El Arroyo-Corinto y Gallinazo-Río Blanco, de oeste a este.

Las tres zonas de cizalla tienen condiciones cinemáticas y deformativas similares; hay desarrollo de zonas de deformación miloníticas anastomosadas y a veces subparalelas, que rodean bloques de rocas poco deformadas o sin deformación por cizalla. A escala microscópica las milonitas presentan bandas de ultramilonitas dentro de protomilonitas y milonita.

Las orientaciones de las zonas de cizalla son muy similares, con rumbos que varían entre N-S y NNE-SSW, y hacia el SE buzamientos de intermedios a altos, los cuales pueden llegar a ser subverticales. Los indicadores cinemáticos analizados a escalas mesoscópica y microscópica permiten deducir movimientos dextrales subhorizontales, dextrales inversos e inversos. Las 
lineaciones de alargamiento de minerales tienen disposiciones que varían entre subhorizontales a subverticales, pasando por oblicuos, con vergencia al SW. Estas combinaciones de movimientos se pueden desarrollar en zonas influenciadas por transpresión y consecuente deformación particionada, la cual se caracteriza por una componente subhorizontal de cizalla simple y una componente subhorizontal ortogonal de cizalla pura, que produce aplastamiento y elongación subvertical.

Las milonitas desarrolladas en las zonas de cizalla se clasifican como milonitas de bajo grado, lo cual se puede deducir a partir de las microestructuras de deformación determinadas a escala microscópica.

Se sugiere que la edad de las zonas miloníticas está en el rango del Cretácico Tardío por comparación con zonas similares dentro del complejo (Vinasco and Cordani, 2012). La existencia de zonas deformadas dentro del Complejo Quebradagrande con componentes transpresivos dextrales indica disgregación de bloques y trasporte de magnitud desconocida desde el sur. Se plantea que estos fenómenos fueron el resultado de la colisión oblicua de la Placa Caribe contra el norte de Sudamérica durante el Cretácico Tardío.

\section{AGRADECIMIENTOS}

Los autores expresan sus agradecimientos a la Vicerrectoría de Investigaciones y Posgrados de la Universidad de Caldas por la financiación brindada para el desarrollo de esta investigación. A los estudiantes Sergio Martínez, Daniel Rodríguez, Santiago Palacios y Julián Morales, del programa de Geología por el acompañamiento en las labores de campo. A los evaluadores del presente trabajo, doctores Javier Escuder-Viruete y Fernando Hongn, cuyas valiosas observaciones y correcciones ayudaron a mejorar este manuscrito. Al geólogo Sebastián Vargas y al doctor Warren Dewhurst por la traducción del resumen.

\section{REFERENCIAS}

Álvarez, J. 1983. Geología de la Cordillera Central y el Occidente Colombiano y petroquimica de los intrusivos granitoides Meso-Cenozoicos. Boletín Geológico, INGEOMINAS, 26: 1-175.

Arancibia, G., Cembrano, J., y Lavenu, A. 1999. Transpresión dextral y partición de la deformación en la Zona de Falla Liquiñe-Ofqui, Aisén, Chile (44-45 $\mathrm{S})$. Revista Geológica de Chile, 26. 3-22.
Barrero, D. 1979. Geology of the central Western Cordillera, west of Buga and Roldanillo, Colombia. INGEOMINAS. Universidad Nacional. Medellín, pp. 1-75.

Fossen, H. 2010. Structural Geology. Cambridge University Press. New York, 463p.

Giunta, G., Beccaluva, L., Coltorti, M., Siena, F., and Vaccaro, C. 2002. The southern margin of the Caribbean Plate in Venezuela: tectono-magmatic setting of the ophiolitic units and kinematic evolution. Lithos, 63: 19-40.

Gómez-Cruz, A.d.J., Moreno-Sánchez, M., y Pardo, A. 1995. Edad y origen del "complejo metasedimentario Aranzazu-Manizales" en los alrededores de Manizales (departamento de Caldas, Colombia). Geología Colombiana, 19: 83-93.

González, H. 1977. Conceptos de metamorfismo dinámico y su aplicación a la zona de falla de Romeral. Ciencias de la Tierra, 2: 81-106.

Grohmann, C.H., and Campanha, G.A.C. 2010. OpenStereo: open source, cross-platform software for structural geology analysis. AGU 2010 Fall Meeting, San Francisco, United States of America, 35p.

Hincapié, G.J., Toro-Toro, L.M., y Ossa, C.A. 2010. Análisis cinemático y deformativo de los Metagabros del Río Olivares sector NNW de Manizales (Caldas). Boletín de Geología, 32: 85-92.

Martens, K.U., Restrepo, J.J., and Ordoñez-Carmona, O. 2014. The Tahami and Anaconia Terranes of the Colombian Andes: Missing links between the South American and Mexican Gondwana margins. The Journal of Geology, 122: 507-530.

Massey, M.A., and Moecher, D.P. 2013. Transpression, extrusion, partitioning, and lateral escape in the middle crust: significance of structures, fabrics, kinematics, and geochronology in the Bronson Hill zone, southern New England. Journal of Structural Geology, 55: 62-78.

Maya, M., y González, H. 1995. Unidades litodémicas en la Cordillera Central de Colombia. Boletín Geológico, INGEOMINAS, 35: 43-57.

Moreno-Sánchez, M., Gómez-Cruz, A.d.J., y ToroToro, L.M. 2008. Proveniencia del material clástico del Complejo Quebradagrande y su relación con los complejos estructurales adyacentes. Ciencias de la Tierra, 22: 27-38. 
Moreno-Sánchez, M., and Pardo-Trujillo, A. 2003. Stratigraphical and sedimentological constrains on western Colombia: implications on the evolution of the Caribbean Plate. In: Bartolini, C., Buffler, R.T., and Blickwede, J.F. The Circum-Gulf of Mexico and the Caribbean: hydrocarbon habitats, basin formation, and plate tectonics. American Association of Petroleum Geologist, Memoir 79: 891-924.

Mosquera, D., Marín, P., Vesga, C., y Gonzáles, H. 1998. Geología de la plancha 206, Manizales, INGEOMINAS.

Nivia, A., Marriner, G.F., Kerr, A.C., and Tarney, J. 2006. The Quebradagrande Complex: a Lower Cretaceous ensialic marginal basin in the Central Cordillera of the Colombian Andes. Journal of South American Earth Sciences, 21: 423-436.

Passchier, C.W., and Trouw, R.A.J. 2005. Microtectonics. Springer. Berlin, 366p.

Pindell, J.L. 1993. Regional synopsis of Gulf of Mexico and Caribbean evolution. En Pindell, J.L., and Perkins, R. 13th annual research conference proceedings, Mesozoic and Early Cenozoic development of the Gulf of Mexico and Caribbean Region, Volume Gulf Coast Section. Society of Economic Paleontologist and Mineralologist Foundation. Houston, pp. 251-274.

Pindell, J.L. 1994. Evolution of the Gulf of Mexico and the Caribbean. In: Caribbean Geology: an introduction (S.K., D., and Jackson, T.A., Eds.). Kingston, Jamaica, University of the West Indies Publishers Association/ University of the West Indies Press, pp. 13-39.

Pindell, J.L. 1997. Tectonic evolution and stratigraphic development of northern South America. VI simposio Bolivariano de Exploración Petrolera en las Cuencas Subandinas, Vol. 2., Cartagena, Colombia.

Pindell, J.L., Higgs, R., and Dewey, J. 1998. Cenozoic palinspastic reconstruction, paleogeographic evolution and Hydrocarbon setting of the northern Margin of South America. In: Pindell, J.L., and Drake, C.L. Paleogeographic Evoloution and Non-Glacial Eustasy, Northern South America. Society of Economic Petrologists and Mineralogists Special Publication, 58: 45-85.

Pindell, J.L., and Kennan, L. 2009. Tectonic evolution of the Gulf of Mexico, Caribbean and northern South America in the mantle reference frame: an update. Geological Society, London, Special Publications, 328(1): 1-55.
Restrepo, J.J., and Toussaint, J.F. 1988. Terranes and continental accretion in the Colombian Andes. Episodes, 11: 189-193.

Sibson, R.H. 1977. Fault rocks and fault mechanisms. Journal of the Geological Society of London, 133: 191213.

Tamayo, J., y Correa, V. 2010. Petrografía y datación de circones detríticos en las facies cuarzosas del complejo Quebradagrande (Cretácico Inferior) de la Cordillera Central. Universidad de Caldas, Manizales, Colombia.

Toro-Toro, L.M., Hincapié, G.J., y Meza, C.A. 2010. Petrografía y geoquímica de los Metagabros del Río Olivares sector NNW de Manizales (Caldas). Boletín de Geología, 32: 73-83.

Trouw, R., Passchier, C.W., and Wiersma, D.J. 2010. Atlas of mylonites and related microstructures. SpringerVerlag. Berlin, 322p.

Vinasco, C.J., and Cordani, U. 2012. Reactivation episodes of the Romeral Fault System in the northwestern part of Central Andes, Colombia, through $39 \mathrm{Ar}$ and K-40 Ar results. Ciencias de la Tierra, 32: 111-124.

Trabajo recibido: abril 20 de 2016

Trabajo aceptado: septiembre 9 de 2016

Manuscrito publicado en internet: septiembre 12 de 2016 